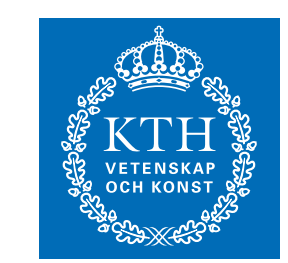

KTH Electrical Engineering

\title{
Exploiting Long-term Statistics in Spatially Correlated Multi-user MIMO Systems with Quantized Channel Norm Feedback
}

\author{
Proceedings of IEEE International Conference on \\ Acoustics, Speech, and Signal Processing (ICASSP)
}

March 30 - April 4, Las Vegas, USA, 2008

(C) 2008 IEEE. Published in the IEEE 2008 International Conference on Acoustics, Speech, and Signal Processing (ICASSP 2008), scheduled for March 30 - April 4, 2008 in Las Vegas, Nevada, U.S.A. Personal use of this material is permitted.

However, permission to reprint/republish this material for advertising or promotional purposes or for creating new collective works for resale or redistribution to servers or lists, or to reuse any copyrighted component of this work in other works, must be obtained from the IEEE. Contact: Manager, Copyrights and Permissions / IEEE Service Center / 445 Hoes Lane / P.O. Box 1331 / Piscataway, NJ 08855-1331, USA. Telephone: + Intl. 908-562-3966.

EMIL BJÖRNSON AND BJÖRN OTTERSTEN

Stockholm 2008

\author{
KTH Royal Institute of Technology \\ ACCESS Linnaeus Center \\ Signal Processing Lab
}

IR-EE-SB 2008:007, Revised version with minor corrections 


\title{
EXPLOITING LONG-TERM STATISTICS IN SPATIALLY CORRELATED MULTI-USER MIMO SYSTEMS WITH QUANTIZED CHANNEL NORM FEEDBACK
}

\author{
Emil Björnson and Björn Ottersten \\ ACCESS Linnaeus Center, Signal Processing Lab, Royal Institute of Technology (KTH) \\ SE-100 44 Stockholm, Sweden (\{emil.bjornson,bjorn.ottersten\} @ee.kth.se)
}

\begin{abstract}
In wireless multiple antenna and multi-user systems, the spatial dimensions may be exploited to increase the performance by means of antenna gain, spatial diversity, and multi-user diversity. A limiting factor in such systems is the channel information required by the transmitter to control the intra-cell interference.

Herein, the properties of spatially correlated channels with longterm statistical information at the transmitter and fixed-rate feedback of the quantized Euclidean channel norm are analyzed using a spectral subspace decomposition framework. A spatial division multiple access scheme is proposed with interference suppression at the receiver and joint scheduling and zero-forcing beamforming at the transmitter. Closed-form expressions for first and second order moments of the feedback conditional channel statistics are derived. It is shown that only a few bits of feedback are required to achieve reliable rate estimation and weighted sum-rate maximization.
\end{abstract}

Index Terms - Array signal processing, Feedback, MIMO systems, Mobile communication, Spatial division multiple access

\section{INTRODUCTION}

The spatial dimensions of a multiple-input multiple-output (MIMO) wireless system can be utilized to increase the performance. When full channel-state information (CSI) is present and the scattering is sufficiently rich, the channel capacity increases linearly with the number of spatial subchannels [1]. The amount of feedback needed to achieve full CSI at the transmitter is however prohibitive in many realistic scenarios. When only long-term statistics and a limited amount of feedback information is available at the transmitter, the spatial dimensions can be exploited using spatial division multiple access (SDMA) [2]. This technique is used in this paper in the downlink of a multi-user MIMO system with spatially correlated fading.

Several approaches have been proposed to implement SDMA in systems with limited feedback-for example, opportunistic beamforming [3] and feedback supported eigenbeamforming [4]. In multi-user opportunistic beamforming, the base station randomly chooses a set of orthogonal beamforming vectors (i.e., directions in which data may be transmitted to different users). Each user feeds back the index of the beam that provides the largest signalto-interference-and-noise ratio (SINR) and the corresponding value of the SINR. When the number of users is large, it is likely that there for each beam exist users that experience particularly strong SINRs.

The SDMA systems analyzed in [4] follow another approach, namely that each single-antenna user feeds back the norm of its

This work is supported in part by the FP6 project Cooperative and Opportunistic Communications in Wireless Networks (COOPCOM), Project Number: FP6-033533. channel (before determining the transmit beamformers). This information is sufficient to perform joint scheduling and beamforming in a spatially correlated channel. Minimum mean square error (MMSE) estimates of signal and interference powers are calculated using [5].

Herein, the feedback supported eigenbeamforming approach in $[4,5]$ is generalized to a MIMO system with feedback of the $L$ bit quantized Euclidean channel norm. A communication model is proposed where the transmissions take place over the statistically strongest subchannel and where receive beamformers are used to suppress the most interfering subchannels over which no signal power is transmitted. Joint scheduling and zero-forcing beamforming is utilized to almost completely suppress the intra-cell interference. Finally, it is shown in simulations that the proposed method outperforms opportunistic beamforming.

\section{SYSTEM MODEL}

The system model considers the downlink of a macro-cellular environment with a single elevated base station and several mobile users. The base station is exposed to little or no local scattering and is equipped with an array of $n_{T}$ antennas, while each terminal is surrounded by rich local scattering and is equipped with an array of $n_{R}$ antennas. The symbol-sampled complex baseband equivalent of the narrowband flat fading channel to user $k$ is represented by

$$
\mathbf{H}_{k}=\left[\mathbf{h}_{k, 1} \ldots \mathbf{h}_{k, n_{R}}\right]^{H} \in \mathbb{C}^{n_{R} \times n_{T}},
$$

where $[\cdot]^{H}$ denotes the conjugate transpose. The elements of the channel matrix $\mathbf{H}_{k}$ to user $k$ are modeled as Rayleigh fading. The rows of $\mathbf{H}_{k}$ are independent and identically distributed as $\mathbf{h}_{k, n} \in$ $\mathcal{C N}\left(\mathbf{0}, \mathbf{R}_{k}\right), n=1, \ldots, n_{R}$. Here, $\mathbf{R}_{k} \in \mathbb{C}^{n_{T} \times n_{T}}$ is a Hermitian, positive definite covariance matrix. These assumptions can be relaxed leading to slight differences in the analysis. Under the current environment assumptions, the covariance matrix $\mathbf{R}_{k}$ will be dominated by one or a few eigenmodes - that is, there is significant spatial correlation [6]. The received vector $\mathbf{y}_{k}(t)$ of user $k$ is modeled as

$$
\mathbf{y}_{k}(t)=\mathbf{V}_{k}^{H} \mathbf{H}_{k} \mathbf{x}(t)+\mathbf{n}_{k}(t),
$$

where $\mathbf{x}(t)$ is the transmitted signal vector, $\mathbf{n}_{k}(t) \in \mathcal{C N}\left(\mathbf{0}, \sigma_{k}^{2} \mathbf{I}\right)$ is additive white noise, and $\mathbf{V}_{k} \in \mathbb{C}^{n_{R} \times n_{R}-n_{I}}$ is the receive beamforming matrix ( $n_{I}$ is defined in the next section). The system model depends on three different time scales. The index $t \in \mathbb{Z}$ denotes the symbol slot on which scale the noise is a white process. The multipath propagation is modeled as quasi-static block fading; that is, the channel realization is constant for a block of symbols and for the next block the channel is modeled as independent. The statistics change even more slowly due to large scale variations, thus the base station is assumed to track the current $\mathbf{R}_{k}$ and $\sigma_{k}^{2}$ perfectly (using the reverse link channel estimates or negligible long-term overhead). The current channel matrix realization $\mathbf{H}_{k}$ is only known to user $k$. 


\section{PROPOSED COMMUNICATION SYSTEM}

In this section we propose a spectral subspace decomposition of the channel statistics (similar to [4]) that is used to characterize the subspaces that are useful for transmission and those that play an essential role for the interference. Let $\mathbf{R}_{k}=\mathbf{U}_{k} \widetilde{\boldsymbol{\Lambda}}_{k} \mathbf{U}_{k}^{H}$ be the eigenvalue decomposition of the covariance matrix of user $k$. Assume that the eigenvalues of $\mathbf{R}_{k}$ are distinct. Then, without loss of generality, we can assume that $\widetilde{\boldsymbol{\Lambda}}_{k}=\operatorname{diag}\left(\lambda_{1}^{(k)}, \ldots, \lambda_{n_{T}}^{(k)}\right)$ with the eigenvalues $\lambda_{i}^{(k)}$ ordered decreasingly. The eigenvectors are given by the corresponding columns of the unitary matrix $\mathbf{U}_{k}$. The assumption of spatial correlation motivates the subspace partitioning

$$
\mathbf{R}_{k}=\left[\mathbf{u}_{k}^{(D)} \mathbf{U}_{k}^{(I)} \mathbf{U}_{k}^{(0)}\right] \widetilde{\boldsymbol{\Lambda}}_{k}\left[\mathbf{u}_{k}^{(D)} \mathbf{U}_{k}^{(I)} \mathbf{U}_{k}^{(0)}\right]^{H},
$$

where $\mathbf{u}_{k}^{(D)} \in \mathbb{C}^{n_{T} \times 1}$ spans the subspace associated with the largest and thereby dominating eigenvalue. This subspace will later be used for transmission. Similarly, $\mathbf{U}_{k}^{(I)} \in \mathbb{C}^{n_{T} \times n_{I}}$ spans the subspace associated with the $n_{I}$ following eigenvalues which are non-negligible compared to the dominating eigenvalue. Hence, the user will be sensitive to interference in this subspace and may require interference suppression. Finally, $\mathbf{U}_{k}^{(0)} \in \mathbb{C}^{n_{T} \times n_{0}}$ spans the subspace of the remaining $n_{0}=n_{T}-n_{I}-1$ eigenmodes. These eigenvalues are typically close to zero, thus the user can ignore interference in this subspace. The optimal dimension, $n_{I}$, of $\mathbf{U}_{k}^{(I)}$ depends on, for example, the channel statistics, the number of users, and the operating SINR. Herein, it is viewed as a design parameter that can be used to optimize the performance for each given system. The only requirement herein is that $n_{I} \leq n_{R}-1$, since we will use the receive beamformer to cancel the interference transmitted over $\mathbf{U}_{k}^{(I)}$.

\subsection{Transmit Beamforming and SINR}

In order to maximize the received signal power, the transmit beamformer $\mathbf{w}_{k} \in \mathbb{C}^{n_{T}}$ of user $k$ should be chosen with a large component within the dominating subspace (i.e., $\mathbf{w}_{k}=\mathbf{u}_{k}^{(D)}$ ). Since we have an SDMA system, the base station should also consider the interference that the transmission to user $k$ inflicts on all simultaneous co-channel users when choosing the beamformer. In Section 5 we propose the use of a greedy user selection with zero-forcing beamforming to solve the problem of allocating transmission resources to an appropriate set of users with suitable beamformers. Let $\mathcal{S}$ denote the set of users scheduled for transmission in symbol slot $t, p_{k}$ the allocated transmission power, and $s_{k}(t)$ the symbol intended for user $k \in \mathcal{S}$, then the transmitted signal $\mathbf{x}(t)$ in (2) is defined as

$$
\mathbf{x}(t)=\sum_{k \in \mathcal{S}} \sqrt{p_{k}} \mathbf{w}_{k} s_{k}(t)
$$

The channel quality when considering interference can be characterized by the SINR. Let $\|\cdot\|$ denote the Euclidean norm. Then, the instantaneous SINR for each symbol transmitted to user $k$, ignoring inter-cell interference, is obtained by combining (2) and (4):

$$
\operatorname{SINR}_{k}=\frac{p_{k}\left\|\mathbf{V}_{k}^{H} \mathbf{H}_{k} \mathbf{w}_{k}\right\|^{2}}{\sum_{i \in \mathcal{S} \backslash\{k\}} p_{i}\left\|\mathbf{V}_{k}^{H} \mathbf{H}_{k} \mathbf{w}_{i}\right\|^{2}+\sigma_{k}^{2}} .
$$

\subsection{Receive Beamforming}

A receive beamformer is proposed that exploits that the receiver has knowledge of the channel realization $\mathbf{H}_{k}$ while the transmitter only has long-term statistical information. Our approach aims at providing reliable SINR estimation at the transmitter and is not claimed to maximize the SINR. The purpose of the receive beamformer is to increase the SINR by suppressing the interference while maintaining most of the signal power. The receiver has no information regarding the other users, but the subspace partition in (3) suggests that the interference mostly originates from interfering transmissions over the subspaces $\mathbf{u}_{k}^{(D)}$ and $\mathbf{U}_{k}^{(I)}$. Since the exact signal and interference structure is unknown to the receiver, it cannot selectively suppress the interference in $\mathbf{u}_{k}^{(D)}$. Therefore, $\mathbf{U}_{k}^{(I)}$ is more suitable to interference cancellation. This subspace is not used for signalling and hence we propose that the receive beamformer $\mathbf{V}_{k}$ should be used to cancel all power transmitted in $\mathbf{H}_{k} \mathbf{U}_{k}^{(I)}$ by choosing it as

$$
\mathbf{V}_{k}=\operatorname{null}\left(\left(\mathbf{H}_{k} \mathbf{U}_{k}^{(I)}\right)^{H}\right) \in \mathbb{C}^{n_{R} \times n_{R}-n_{I}},
$$

where null( $\cdot)$ gives an orthonormal basis to the right null space and can be calculated using the singular value decomposition. Let $\overline{\mathbf{H}}_{k}=\mathbf{V}_{k}^{H} \mathbf{H}_{k}=\left[\overline{\mathbf{h}}_{k, 1}, \ldots, \overline{\mathbf{h}}_{k, n_{R}-n_{I}}\right]^{H}$ and observe that $\mathbf{V}_{k}$ is determined using only the channel realization within the interference sensitive subspace. Hence, the experienced channel $\overline{\mathbf{H}}_{k}$ will still be Rayleigh fading. The transmitter can therefore exploit that $\overline{\mathbf{h}}_{k, n} \in \mathcal{C N}\left(\mathbf{0}, \mathbf{Q}_{k}\right)$, for $n=1, \ldots, n_{R}-n_{I}$, where the covariance matrix is defined by the eigenvalue decomposition $\mathbf{Q}_{k}=\mathbf{U}_{k} \boldsymbol{\Lambda}_{k} \mathbf{U}_{k}^{H}$ with $\mathbf{U}_{k}$ as the partioned matrix in (3) and

$$
\boldsymbol{\Lambda}_{k}=\operatorname{diag}\left(\lambda_{1}^{(k)}, 0, \ldots, 0, \lambda_{n_{I}+2}^{(k)}, \ldots, \lambda_{n_{T}}^{(k)}\right) .
$$

Hence, the receive beamformer will transform the MIMO channel into a channel that essentially has rank one and is aligned with $\mathbf{u}_{k}^{(D)}$.

\subsection{Feedback and Rate Estimation}

In order to ensure reliable communication to each scheduled user, no user should be assigned a rate exceeding its outage capacity. Since the capacity is a function of the instantaneous SINR it is important for the transmitter not to overestimate the SINR, given in (5), with a probability larger than the acceptable outage probability.

The MMSE estimates of the instantaneous signal and interference power can be calculated using the channel statistics. The estimates can be improved significantly by utilizing feedback of the channel norm-especially when the norm is strong (relative to its mean value) [5] and strong users are good candidates for scheduling. Herein, we consider a system where each user $k$ feeds back a quantized variable, $\rho_{k}$, representing an interval of the squared $\mathrm{Eu}-$ clidean norm $\left\|\mathbf{V}_{k}^{H} \mathbf{H}_{k}\right\|^{2}$, through an error-free feedback channel. This variable is properly defined in Section 4 , where it is used to derive moments of the conditional channel statistics.

Let $E\{\cdot\}$ and $D\{\cdot\}$ denote the mean value and square root of the variance, respectively, and let $\mathcal{S}_{\tilde{k}} \triangleq \mathcal{S} \backslash\{k\}$ and $\boldsymbol{\rho}_{\tilde{k}} \triangleq\left\{\rho_{i}\right\}_{i \in \mathcal{S} \backslash\{k\}}$. Similar to [4], the SINR can then be estimated pessimistically (with the design parameter $\alpha$ to fulfill the outage probability) as

$\widehat{\operatorname{SINR}}_{k}=\frac{p_{k}\left[E\left\{\left\|\overline{\mathbf{H}}_{k} \mathbf{w}_{k}\right\|^{2} \mid \rho_{k}\right\}-\alpha D\left\{\left\|\overline{\mathbf{H}}_{k} \mathbf{w}_{k}\right\|^{2} \mid \rho_{k}\right\}\right]}{\sum_{i \in \mathcal{S}_{\tilde{k}}} p_{i} E\left\{\left\|\overline{\mathbf{H}}_{k} \mathbf{w}_{i}\right\|^{2} \mid \rho_{k}\right\}+\alpha D\left\{\sum_{i \in \mathcal{S}_{\tilde{k}}}\left\|\overline{\mathbf{H}}_{k} \mathbf{w}_{i}\right\|^{2} \mid \boldsymbol{\rho}_{\tilde{k}}\right\}+\sigma_{k}^{2}}$

and utilized to calculate the supported rate using the rate function $r_{k}=r\left(\mathrm{SINR}_{k}\right)$, which is assumed to be non-decreasing. The Shannon capacity $r(x)=\log _{2}(1+x)$ will be used as rate function in the simulations, but nothing else in the paper is restricted to this choice.

\subsection{System Operation}

The cyclic system operation is shown in Fig. 1. Recall that the channel is modeled as block fading. The cycle starts in the beginning of a 


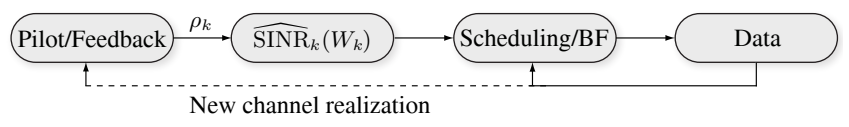

Fig. 1. Schematic system operation at the base station.

block and orthogonal pilot sequences are transmitted by the base station, which allows each user to estimate its current channel realization and use it to determine its receive beamforming matrix $\mathbf{V}_{k}$ using (6) and calculate the squared channel norm $\left\|\mathbf{V}_{k}^{H} \mathbf{H}_{k}\right\|^{2}$. Each user feeds back its $L$-bit quantization $\rho_{k}$ of the squared channel norm. The base station combines the feedback with the long-term statistics to estimate the SINR as a function of the transmit beamformers. The scheduler uses these estimates to iteratively allocate resources to appropriate sets of users. When a block with a new channel realization begins, the cycle starts all over again.

\section{4. $L$-BIT QUANTIZED CHANNEL NORM FEEDBACK}

It has been shown under various conditions $[4,5,7]$ how the channel statistics can be combined with the Euclidean norm of the channel matrix to perform beamforming and acquire conditional channel information at the transmitter. In general, it will however be impossible to feed back the real-valued channel norm using a limited number of bits. This paper therefore analyzes the case when user $k$ feeds back $\rho_{k}$, which is an $L$-bit quantization of the squared channel norm $\left\|\overline{\mathbf{H}}_{k}\right\|^{2}$ representing a certain interval of the value.

Next, we discuss how the interval boundaries can be chosen and the SINR estimated at the transmitter, but only for $n_{I}=n_{R}-1$ (i.e., $\overline{\mathbf{H}}_{k}=\overline{\mathbf{h}}_{k, 1}^{H}$ ) due to space limitation. The user indices will be dropped in this section for brevity. First, we consider the distribution of $\|\overline{\mathbf{H}}\|^{2}$. Let $\overline{\mathbf{v}}$ be the vector of independent variables that satisfy $\overline{\mathbf{v}}=\mathbf{U}^{H} \overline{\mathbf{h}}$. Observe that $\|\overline{\mathbf{v}}\|^{2}=\|\overline{\mathbf{h}}\|^{2}$ and $\overline{\mathbf{v}} \in \mathcal{C N}(\mathbf{0}, \boldsymbol{\Lambda})$. Let $\Omega$ be the index set of all non-zero eigenvalues of $\boldsymbol{\Lambda}$. The CDF of $\|\overline{\mathbf{h}}\|^{2}$ is [5]

$$
F_{\|\overline{\mathbf{h}}\|^{2}}(\varrho)=1-\sum_{j \in \Omega} \frac{e^{-\frac{\varrho}{\lambda_{j}}}}{\prod_{i \in \Omega \backslash\{j\}}\left(1-\frac{\lambda_{i}}{\lambda_{j}}\right)} .
$$

The range of $\|\overline{\mathbf{h}}\|^{2} \in[0, \infty)$ should be divided into $2^{L}$ disjoint intervals, such that both the transmitter and the receiver knows that $\rho$ means that $A_{\rho} \leq\|\overline{\mathbf{h}}\|^{2}<B_{\rho}$. Since the scheduler seeks to allocate users with strong channel realizations, we propose that the first interval should contain $\|\overline{\mathbf{h}}\|^{2}<F_{\|\overline{\mathbf{h}}\|^{2}}^{-1}(C)$, where $F_{\|\overline{\mathbf{h}}\|^{2}}^{-1}(\cdot)$ denotes the inverse of the $\mathrm{CDF}$ and $C$ is a design parameter. $C$ is chosen such that sufficiently many users are likely to have a squared norm outside the first interval to perform efficient scheduling. The remaining probability density is divided into intervals of equal probability. Hence, if $\rho_{k} \in\left\{0, \ldots, 2^{L}-1\right\}$ then $A_{0}=0, B_{2^{L}-1}=\infty$, and

$$
A_{j+1}=B_{j}=F_{\|\overline{\mathbf{h}}\|^{2}}^{-1}\left(C+\frac{1-C}{2^{L}-1} j\right), j=0, \ldots, 2^{L}-2 .
$$

No closed-form expression is provided for the bijective and nondecreasing function $F_{\|\overline{\mathbf{h}}\|^{2}}^{-1}(\cdot)$, but estimates of $A_{j}$ and $B_{j}$ can be calculated efficiently using a line search.

\subsection{Calculating the First and Second Order Moments}

Theorem 1 gives closed-form expressions of the first and second order moments of the channel statistics, conditioned on the quantized feedback parameter $\rho_{k}$, for the special case when the covariance matrix is diagonal and has distinct eigenvalues. Corollary 1 shows how these results are generalized to the channel conditions considered in this paper ( still $n_{I}=n_{R}-1$ ). These results can be used directly to calculate the SINR estimate in (8) as a function of the transmit beamformer, which corresponds to the second box in the system operation shown in Fig. 1. Observe that the quantization and the expressions in Theorem 1 only depend on the long-term statistics and therefore need to be recalculated only at the rate the statistics change.

Theorem 1. Let $\mathbf{v}=\left[v_{1}, \ldots, v_{N}\right]^{T} \in \mathcal{C N}(\mathbf{0}, \boldsymbol{\Lambda})$, with the covariance matrix $\boldsymbol{\Lambda}=\operatorname{diag}\left\{\lambda_{1}, \ldots, \lambda_{N}\right\}$ where all $\lambda_{i}$ are distinct and strictly positive. Let the variable $\rho$ contain the information that $A_{\rho} \leq\|\mathbf{v}\|^{2}<B_{\rho}$. Let $n \neq l, g_{\rho}=\sum_{k=1}^{N} \frac{e^{-A_{\rho} / \lambda_{k}}-e^{-B_{\rho} / \lambda_{k}}}{\prod_{i \neq k}\left(1-\lambda_{i} / \lambda_{k}\right)}$, $S_{j}^{1}=\sum_{i \neq j} \frac{\lambda_{i}}{1-\lambda_{i} / \lambda_{j}}$, and $S_{j}^{2}=\sum_{i \neq j} \frac{\lambda_{i}^{2}}{\left(1-\lambda_{i} / \lambda_{j}\right)^{2}}$. Then

$$
\begin{aligned}
E\left\{\left|v_{l}\right|^{2} \mid \rho\right\} & =\frac{1}{g_{\rho}}\left[\frac{\left(A_{\rho}+\lambda_{l}\right) e^{-\frac{A_{\rho}}{\lambda_{l}}}-\left(B_{\rho}+\lambda_{l}\right) e^{-\frac{B_{\rho}}{\lambda_{l}}}}{\prod_{i \neq l}\left(1-\frac{\lambda_{i}}{\lambda_{l}}\right)}\right. \\
& \left.+\sum_{k \neq l} \frac{\lambda_{l}\left(e^{-\frac{A_{\rho}}{\lambda_{l}}}-e^{-\frac{B_{\rho}}{\lambda_{l}}}\right)-\lambda_{k}\left(e^{-\frac{A_{\rho}}{\lambda_{k}}}-e^{-\frac{B_{\rho}}{\lambda_{k}}}\right)}{\left(1-\frac{\lambda_{k}}{\lambda_{l}}\right) \prod_{i \neq k}\left(1-\frac{\lambda_{i}}{\lambda_{k}}\right)}\right]
\end{aligned}
$$

$E\left\{\left|v_{l}\right|^{4} \mid \rho\right\}=\frac{1}{g_{\rho}}\left[\frac{\left(A_{\rho}+\lambda_{l}-S_{l}^{1}\right)^{2} e^{-\frac{A_{\rho}}{\lambda_{l}}}-\left(B_{\rho}+\lambda_{l}-S_{l}^{1}\right)^{2} e^{-\frac{B_{\rho}}{\lambda_{l}}}}{\prod_{i \neq l}\left(1-\frac{\lambda_{i}}{\lambda_{l}}\right)}\right.$ $\left.+\frac{\left(\lambda_{l}^{2}+S_{l}^{2}\right)\left(e^{-\frac{A_{\rho}}{\lambda_{l}}}-e^{-\frac{B_{\rho}}{\lambda_{l}}}\right)}{\prod_{i \neq l}\left(1-\frac{\lambda_{i}}{\lambda_{l}}\right)}+\sum_{k \neq l} \frac{2 \lambda_{k}^{2}\left(e^{-\frac{A_{\rho}}{\lambda_{k}}}-e^{-\frac{B_{\rho}}{\lambda_{k}}}\right)}{\left(1-\frac{\lambda_{k}}{\lambda_{l}}\right)^{2} \prod_{i \neq k}\left(1-\frac{\lambda_{i}}{\lambda_{k}}\right)}\right]$

$E\left\{\left|v_{n}\right|^{2}\left|v_{l}\right|^{2} \mid \rho\right\}=\frac{1}{g_{\rho}}\left[\sum_{k \notin\{n, l\}} \frac{\lambda_{k}^{2}\left(e^{-\frac{A_{\rho}}{\lambda_{k}}}-e^{-\frac{B_{\rho}}{\lambda_{k}}}\right)}{\left(1-\frac{\lambda_{k}}{\lambda_{l}}\right)\left(1-\frac{\lambda_{k}}{\lambda_{n}}\right) \prod_{i \neq k}\left(1-\frac{\lambda_{i}}{\lambda_{k}}\right)}\right.$ $+\frac{\left(\lambda_{n} S_{n}^{1}-\lambda_{n}^{2}+\frac{\lambda_{l} \lambda_{n}}{1-\frac{\lambda_{l}}{\lambda_{n}}}\right)\left(e^{-\frac{A_{\rho}}{\lambda_{n}}}-e^{-\frac{B_{\rho}}{\lambda_{n}}}\right)-\lambda_{n}\left(A_{\rho} e^{-\frac{A_{\rho}}{\lambda_{n}}}-B_{\rho} e^{-\frac{B_{\rho}}{\lambda_{n}}}\right)}{\left(1-\frac{\lambda_{n}}{\lambda_{l}}\right) \prod_{i \neq n}\left(1-\frac{\lambda_{i}}{\lambda_{n}}\right)}$ $\left.+\frac{\left(\lambda_{l} S_{l}^{1}-\lambda_{l}^{2}+\frac{\lambda_{n} \lambda_{l}}{1-\frac{\lambda_{n}}{\lambda_{l}}}\right)\left(e^{-\frac{A_{\rho}}{\lambda_{l}}}-e^{-\frac{B_{\rho}}{\lambda_{l}}}\right)-\lambda_{l}\left(A_{\rho} e^{-\frac{A_{\rho}}{\lambda_{l}}}-B_{\rho} e^{-\frac{B_{\rho}}{\lambda_{l}}}\right)}{\left(1-\frac{\lambda_{l}}{\lambda_{n}}\right) \prod_{i \neq l}\left(1-\frac{\lambda_{i}}{\lambda_{l}}\right)}\right]$

Proof. The proof follows by the law of total probability, using expressions derived in [5]. A special case has been shown in [7].

Corollary 1. Let $\mathbf{h} \in \mathcal{C N}(\mathbf{0}, \mathbf{R})$, where $\mathbf{R}=\mathbf{U} \overline{\mathbf{\Lambda}} \mathbf{U}^{H} \in \mathbb{C}^{N \times N}$ is the eigenvalue decomposition. Let all non-zero eigenvalues $\lambda_{i}$ of $\bar{\Lambda}$ be distinct, positive, and have indices gathered in the set $\Omega$. Let $\mathbf{u}$ and $\widetilde{\mathbf{u}}$ be two complex-valued vectors and define $\mathbf{w}=$ $\left[w_{1}, \ldots, w_{N}\right]^{T}=\mathbf{U}^{H} \mathbf{u}$ and $\widetilde{\mathbf{w}}=\left[\tilde{w}_{1}, \ldots, \tilde{w}_{N}\right]^{T}=\mathbf{U}^{H} \widetilde{\mathbf{u}}$. Let $\rho$ contain the information that $A_{\rho} \leq\|\mathbf{h}\|^{2}<B_{\rho}$. Next, define $\widehat{\Lambda} \in \mathbb{C}^{N \times N}$ and $\mathbf{T} \in \mathbb{C}^{N \times N}$ as

$$
[\widehat{\boldsymbol{\Lambda}}]_{i j}=\left\{\begin{array}{ll}
E\left\{\left|v_{i}\right|^{2} \mid \rho\right\}, & i=j, \\
0, & i \neq j,
\end{array} \quad[\mathbf{T}]_{i j}= \begin{cases}E\left\{\left|v_{i}\right|^{4} \mid \rho\right\}, & i=j, \\
2 E\left\{\left|v_{i}\right|^{2}\left|v_{j}\right|^{2} \mid \rho\right\}, & i \neq j,\end{cases}\right.
$$

where $E\left\{\left|v_{i}\right|^{2} \mid \rho\right\}=E\left\{\left|v_{i}\right|^{4} \mid \rho\right\}=0$ for $i \notin \Omega, E\left\{\left|v_{i}\right|^{2}\left|v_{j}\right|^{2} \mid \rho\right\}=0$ for $i \notin \Omega$ or $j \notin \Omega$ and the other terms are given by Theorem 1 using $\boldsymbol{\Lambda}=\operatorname{diag}\left(\left\{\lambda_{l} ; l \in \Omega\right\}\right)$. Then the following expressions arise:

$$
\begin{aligned}
E\left\{\left|\mathbf{h}^{H} \mathbf{u}\right|^{2} \mid \rho\right\} & =\mathbf{w}^{H} \widehat{\Lambda} \mathbf{w}=\mathbf{u}^{H} \mathbf{U} \widehat{\mathbf{\Lambda}} \mathbf{U}^{H} \mathbf{u}, \\
E\left\{\left\|\mathbf{h}^{H} \mathbf{u}\right\|^{2}\left\|\mathbf{h}^{H} \widetilde{\mathbf{u}}\right\|^{2} \mid \rho\right\} & =\frac{1}{2}\left(\mathbf{p}^{H} \mathbf{T} \widetilde{\mathbf{p}}+\overline{\mathbf{p}}^{H} \mathbf{T} \overline{\mathbf{p}}\right), \\
E\left\{\left\|\mathbf{h}^{H} \mathbf{u}\right\|^{4} \mid \rho\right\} & =\mathbf{p}^{H} \mathbf{T} \mathbf{p},
\end{aligned}
$$


where $\mathbf{p}=\left[\left|w_{1}\right|^{2}, \ldots,\left|w_{N}\right|^{2}\right]^{H}, \widetilde{\mathbf{p}}=\left[\left|\tilde{w}_{1}\right|^{2}, \ldots,\left|\tilde{w}_{N}\right|^{2}\right]^{H}$, and $\overline{\mathbf{p}}=\left[w_{1} \tilde{w}_{1}^{*}, \ldots, w_{N} \tilde{w}_{N}^{*}\right]^{H}$.

\section{JOINT SCHEDULING AND BEAMFORMING}

In order to allocate resources to appropriate sets of users with suitable beamforming matrices, a scheduler needs to be defined. Optimal joint scheduling and beamforming, in the sense of maximizing some weighted sum-rate criterion, is very difficult to achieve and the solution is usually too complex for real-time applications. To show the merits of our communication scheme, we use the suboptimal greedy resource allocation algorithm proposed in [4]. This algorithm is based on maximizing the weighted sum-rate criterion $R_{\Sigma}=\sum_{k \in \mathcal{S}} \beta_{k} r\left(\widehat{\mathrm{SINR}}_{k}\right)$ by choosing the set of scheduled users $\mathcal{S}$ and the beamforming vector $\mathbf{w}_{k}$ for $k \in \mathcal{S}$. The user priority is determined by $\beta_{k}$ and can be used to control the fairness. No limitations are introduced on how the signal power can be allocated.

The algorithm is iterative and searches for the user $k \notin \mathcal{S}$ that would provide the largest increase in $R_{\Sigma}$ if added to $\mathcal{S}$. We add this user to $\mathcal{S}$ and proceed iteratively as long as $|\mathcal{S}|<n_{T}$ and $R_{\Sigma}$ is increasing. The transmit beamformers $\mathbf{w}_{k}$ are derived by estimating the experienced channel $\overline{\mathbf{H}}_{k}$ of user $k$ by $\left(\mathbf{u}_{k}^{(D)}\right)^{H}$ and then performing traditional zero-forcing for systems with single receive antennas.

\section{PERFORMANCE EVALUATION}

The performance of the proposed system is compared to opportunistic beamforming in a circular cell with an eight-antenna uniform circular array (UCA) at the base station and with mobile users equipped with four antennas. The angular spread is 15 degrees as seen from the base station. The power allocation is uniform and gives an average SNR of $10 \mathrm{~dB}$ for a user at the cell edge with a random transmit beamformer. The same simulation assumptions are made as in [7], including proportional fairness weighting [8] on both methods. The quantization intervals have uniform probability. The evaluation consists of 2600 scenarios, each representing a unique random constellation of mobiles with fixed statistics, where the average cell throughput is calculated over 64 blocks with independent channel realizations, each used to schedule symbols in 4 slots. The random beams of the opportunistic beamforming are fixed during each realization, so the feedback is comparable to the proposed system. The outage probability of the proposed system is approximately $5 \%$.

The cumulative distribution functions (CDFs) of the the average cell throughput (over scenarios) is shown in Fig. 2a for a lightly loaded cell ( 8 users) and in Fig. 2b for a heavily loaded cell (32 users). Opportunistic beamforming with unquantized feedback is compared to the proposed beamforming system with $0,1,3$, or 5 bits of feedback or unquantized feedback. Independently of the number of users, only a single bit of feedback is needed to clearly outperform opportunistic beamforming (which requires $\log _{2}\left(n_{T}\right)$ bits just to feed back the index of the best beam). Using only one bit of feedback, the proposed method achieves $48-56 \%$ of the unquantized feedback gain, while three bits gives $90 \%$ and five bits $98-99 \%$.

\section{CONCLUSIONS}

We have proposed a quantized channel norm feedback algorithm for spatially correlated Rayleigh fading MIMO channels. A spectral subspace decomposition framework was used to propose an SDMA system that exploits the spatial dimensions using interference subspace suppression at the receivers and low-complexity greedy joint

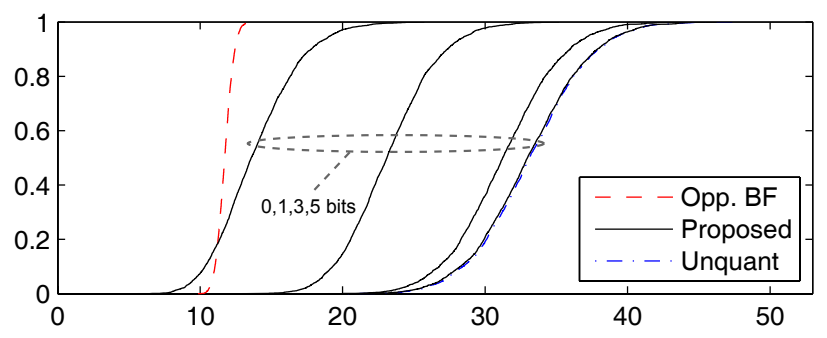

(a) Cell throughput [bits/slot], 8 users.

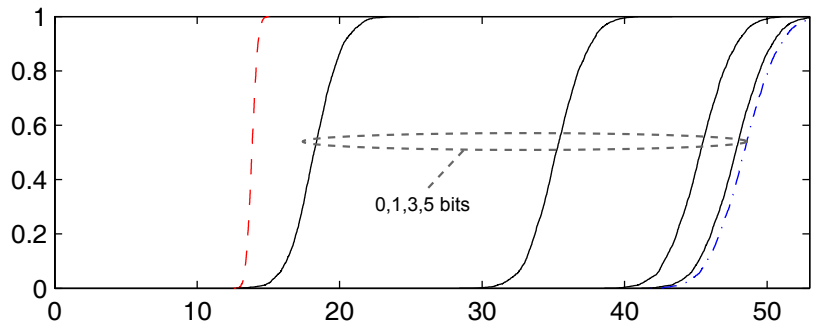

(b) Cell throughput [bits/slot], 32 users.

Fig. 2. The CDFs of the average cell throughput achieved in random scenarios for different number of users and feedback bits.

scheduling and zero-forcing beamforming at the base station. An important feature of the system is that the choice of feedback parameter does not inflict any limitations on the transmit beamforming.

Closed-form expressions for the first and second moments of the quantized channel norm conditional channel statistics were derived and used to achieve reliable rate estimation. Exploiting only feedback information and long-term channel statistics, the proposed system outperforms opportunistic beamforming in the evaluated system setting for any number of feedback bits. Just a few bits are needed to achieve most of the gain compared to unquantized channel norm feedback.

\section{REFERENCES}

[1] E. Telatar, "Capacity of multi-antenna Gaussian channels," Eur. Trans. Telecom., vol. 10, no. 6, pp. 585 - 595, 1999.

[2] M. Schubert and H. Boche, "Solution of the multiuser downlink beamforming problem with individual SINR constraints," IEEE Trans. Vehicular Tech., vol. 53, no. 1, pp. 18-28, 2004.

[3] M. Sharif and B. Hassibi, "On the capacity of MIMO broadcast channels with partial side information," IEEE Trans. on Information Theory, vol. 51, pp. 506-522, 2005.

[4] D. Hammarwall, M. Bengtsson, and B. Ottersten, "Utilizing the spatial information provided by channel norm feedback in SDMA systems," IEEE Trans. on Sig. Proc., Submitted.

[5] D. Hammarwall, M. Bengtsson, and B. Ottersten, "Acquiring partial CSI for spatially selective transmission by instantaneous channel norm feedback," IEEE Trans. on Sig. Proc., To appear.

[6] R. Ertel, P. Cardieri, K. Sowerby, T. Rappaport, and J. Reed, "Overview of spatial channel models for antenna array communication systems," IEEE Pers. Com., pp. 10-22, Feb 1998.

[7] E. Björnson, D. Hammarwall, and B. Ottersten, "Beamforming utilizing channel norm feedback in multiuser MIMO systems," in Proc. IEEE Work. Sig. Process. Adv. in Wireless Com., 2007.

[8] P. Viswanath, D.N.C. Tse, and R. Laroia, "Opportunistic beamforming using dumb antennas," IEEE Trans. on Information Theory, vol. 48, no. 6, pp. 1277-1294, 2002. 